\title{
Algorithm of the target function of time between overhauls taking into account gradual and sudden failure of the locomotive equipment
}

\author{
Anatolii Osyaev ${ }^{1, *}$, Alexandr Rauba ${ }^{2}$, and Alexandr Kosarev $^{3}$ \\ ${ }^{1}$ Russian Open Academy of Transport ROAT (MIIT), Moscow, Russia \\ ${ }^{2}$ Omsk State Transport University, Karl Marx Ave., 35, Omsk, 644046, Russia \\ ${ }^{3}$ Joint-stock company "Scientific and Research Institute of Railway Transport" (JSC "VNIIZhT"), \\ Moscow, Russia
}

\begin{abstract}
The paper considers the principles of technology of scheduled preventive repair of equipment, as well as the principles of repair taking into account the technical condition of locomotive equipment. The main goal of the study is to justify the mechanism for optimizing the repair cycle of locomotives in order to ensure high reliability of the equipment at the lowest costs. For the organization of the strategy of maintenance and repair of the locomotive fleet according to the scheduled preventive repair method and the method taking into account the technical condition, an algorithm for the target function of the time between overhauls has been developed, taking into account the gradual and sudden failures of the locomotive equipment. A description of the model of gradual failures and the target function including the values of mileage and tolerance is given. The results of the study formulated a more universal algorithm of the target function for determining the values of the standard operating time of the assembly unit subjected to both gradual and sudden failures in operation, which includes an analysis of the errors of the first and second kind in diagnosing the object, the probability of replacement and failure detection, and economic indicators. This algorithm is adapted to the repair cycle consisting of three types of repair: the current repair $\mathrm{CR}$, the medium repair $\mathrm{MR}$, and the overall repair OR. It has been established that when optimizing the repair cycle of locomotives while simultaneously taking into account both gradual and sudden equipment failures, a reduction in the number of unscheduled repairs reaches $10-15 \%$, the number of scheduled repairs is reduced by $12-18 \%$.
\end{abstract}

\section{Introduction}

The priority task for the growth of traffic volumes, improving their quality indicators, increasing the reliability and efficiency of the traction complex is the acquisition of locomotives with improved technical characteristics and locomotives of new generation.

Savings of operating costs from the acquisition of such traction rolling stock are achieved by improving the coefficient of technical readiness, reducing the duration and changing the

\footnotetext{
${ }^{*}$ Corresponding author: org@rgotups.ru
} 
structure of the repair cycle, increasing the reliability of locomotives, reducing the specific consumption of fuel and energy resources, and increasing the productivity of locomotive crews in comparison with locomotive analogues.

The main direction of improving the maintenance and repair system ( $M$ and $R$ ) is to increase the reliability of the rolling stock during overall repairs with deep modernization of the units, the introduction of new technological solutions, the non-admittance of equipment operation beyond the established tolerances, and the control of technical condition of the units in operation. At the same time, the intensification of train operation makes increased demands on the reliability of traction rolling stock. Therefore, the management of the technical state of electric locomotives and diesel locomotives in order to maintain it at a given level is one of the most important tasks of the locomotive economy and railway transport in general.

The purpose of this study is the target function of optimizing the repair cycle with the application of criteria for minimum costs and maximum failure-free operation associated with the time between overhauls before the current $\mathrm{CR}$, the medium MR, and the overall OR repair types.

\section{General}

The main factors directly affecting the technical condition (TC) of the locomotive with the currently used scheduled preventive maintenance system (SPM) include the constructive and resource base of units and assemblies of locomotive equipment, the conditions and technology of its operation, and also the frequency, composition, and quality of preventive and recovery work on repairs and maintenance (see Fig. 1).

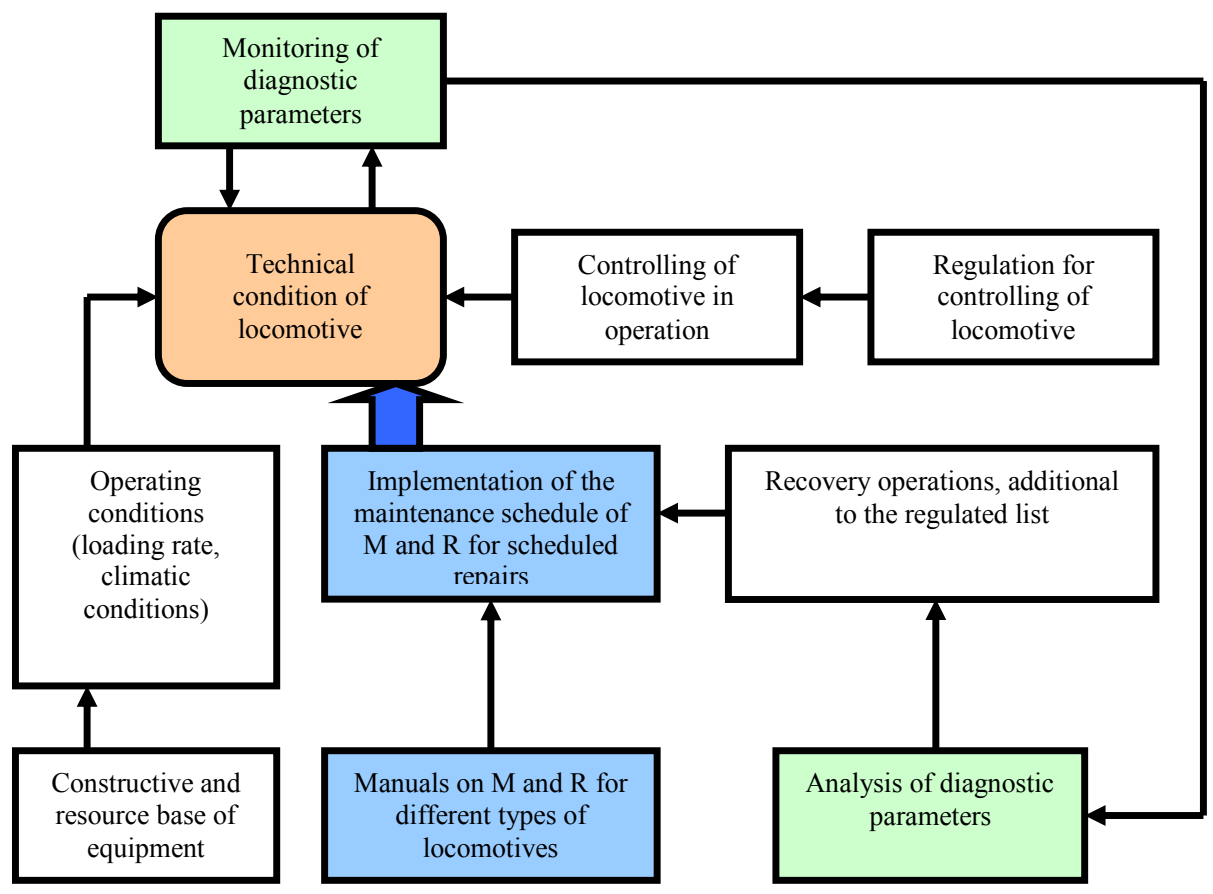

Fig. 1. Structure of the main factors determining the technical condition of the locomotive. 
The purpose of the locomotive is to perform operational work under conditions that meet the requirements for its design and manufacture, and the technology of its operation. Therefore, the number of controllable factors, if it is necessary to improve the $\mathrm{TC}$ and to reduce the costs of maintaining the locomotive, first of all, includes the standardized parameters of the SPM system, which must be adapted to the operation model of the train operation.

Figure 2 shows a generalized scheme of the combined effect of a number of characteristics of the SPM and repair enterprises on the TC of locomotive. At present, the indication of JSC "Russian Railways" determines the types of maintenance and scheduled repair, the average for the railway network standards for their operating time, as well as the regulated lists of works corresponding to them.

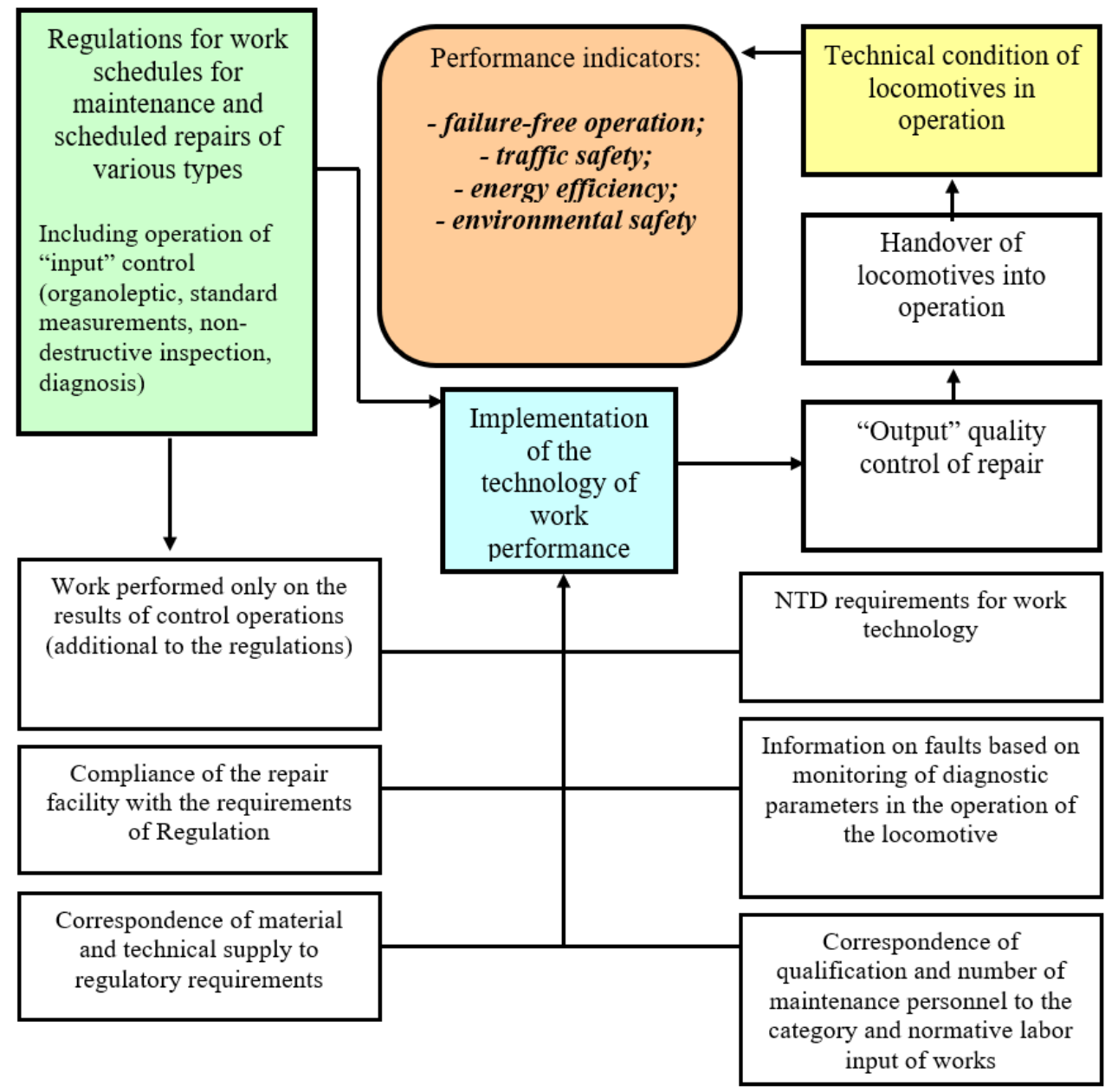

Fig. 2. Scheme of interconnection of types of maintenance and repair of locomotives.

Their real composition is formed from the operations performed regardless of the condition of the assembly units (constituent parts of the locomotive) and the so-called "additional" works, the necessity of which is determined by the results of monitoring the actual state of certain parts, assemblies, units, and apparatuses. 
The reliability of the control, the number of checked elements of the locomotive equipment and the resolving capacity of the "input" control devices used thereby determine the probability of identifying defects whose growing leads to a failure in operation. Thus, the improvement of the monitoring and diagnosing system of locomotive equipment related to maintenance and current repair is one of the ways to control the technical condition of the locomotive.

On the railway network, most locomotive depots are equipped with different diagnostic devices, but the reliability of diagnoses, the number of locomotive units controlled by them, and the reliability of these facilities are not sufficient.

The quality of recovery of the locomotive is significantly influenced by the ability of the repair enterprise to be equipped with technological equipment, materials, spare parts, and labor resources. Planning of these types of maintenance of repair production is based on the corresponding requirements for labor and spare parts use. Their adaptation to the changing operating conditions of the locomotive fleet, to the dynamics of indicators of its operational reliability, of course, should be attributed to the number of measures for managing the TC of electric locomotives and diesel locomotives.

The main characteristics of the locomotive SPM system determining its effectiveness are the normative lists of works subject to mandatory performance at maintenance and repairs of each type, the standards of mileage (running time) before maintenance and repair, as well as the regulated tolerance values for the technical parameters with which the locomotive should come into service after its recovery.

As an alternative to the SPM system for maintenance and repair of locomotives, in some cases, immeasurably better results are obtained by the use of repair technology that takes into account technical condition of the equipment.

With this technology, which involves assessing the technical condition of the equipment without disassembly, in operation modes, it is a question of control by the secondary parameters, and therefore it is logical that these parameters must meet certain requirements. Requirements for them can be formulated, for example, as follows:

- controlled parameters must have a unique quantitative relationship with the primary parameters of the technical condition;

- measurement of parameters should possibly be ensured by simple, portable technical means, which do not require special qualification of personnel;

- technical means must be calibrated when necessary;

- the range of change of controlled parameters during the operation of the equipment from the "good" condition to the "unacceptable" condition should be sufficiently large (the parameter should vary not less than 15-20 times) for timely detection of emerging defects and reliable prediction of the residual resource of the equipment;

- the cost of performing work to control secondary parameters and the time of their implementation should be significantly lower than maintenance time of equipment;

- reliability of control over secondary parameters should not be less than $80 \%$;

- control parameters should be as universal as possible to diagnose identical defects of the same equipment or its components.

Taking into account the further development of computer technology and information and measurement systems, it seems possible to combine the technological process of diagnosis with methods for forecasting the technical condition of rolling stock equipment, i.e. the repair technology that takes into account technical condition of the equipment.

The basis of this approach is technical diagnosis (TD) and prediction of the condition of equipment. Using TD means, continuous or periodic control of technical condition of rolling stock equipment is carried out. Forecasting is performed during continuous control to determine the time during which the operable condition remains, and during periodic control - to determine the time of the next control. 
In terms of the management information system for managing the reliability parameters of the locomotive, an automated locomotive reliability management system (ALRMS) can be noted. The automated locomotive reliability management system (ALRMS) is proposed to be created in a single information space, for which an automated system for monitoring the technical condition of locomotives (USMT) should be created, which will unite all participants of the technological process. The initial information for USMT will be the diagnostic data of the on-board microprocessor locomotive control systems (MCS) and automated systems for technical diagnostics (ASTD), as well as the information systems of JSC "Russian Railways", united by the general concept of ALRMS.

\section{Methods}

The general task of optimizing the operational reliability parameters for the locomotive maintenance and repair system ( $\mathrm{M}$ and $\mathrm{R}$ ) involves the use of a rather complex mathematical apparatus. The model for solving this problem is formed taking into account the following provisions. The minimum value of the specific costs (per unit of operating time) for the technical maintenance of the locomotive is assumed as an optimization criterion. The costs include maintenance costs, planned and unplanned repairs, as well as losses from locomotive down time in the depot (during maintenance and repairs) and from failures during operation. The boundary conditions of the solution are usually the specified maximum permissible values of indicators of the locomotive's damageability and the duration of its temporary suspension from operation for maintenance and repairs.

In general, the following target function is used:

$$
q(L)=\frac{1}{L}\left\{C_{H} \int_{0}^{L} \omega(l) d l+C_{\mathrm{S}}\right\} \longrightarrow \min
$$

where: $q$ - total costs;

$C_{H}$ - the average cost due to the failure of the facility during the time between overhauls;

$C s$ - the average cost of scheduled repairs of the object;

$\boldsymbol{\omega}$ - the failure flow parameter;

$l$ - the current value of the operating time of the object;

$\mathrm{L}$ - variable operating time of the object before scheduled repair.

The main different types of locomotive assembly units are considered as an object in order to establish the optimal value of L, the so-called "local" optimum corresponding to the minimum value of q. Later, the definition of the "global" optimum is being conducted basing on qi,min, where $i$ is the type of the assembly unit, for the whole set of locomotive equipment.

To date, there has been no practical method for preventing sudden failures. In this regard, a fairly simple method based on estimating the distribution of the operating time to failure with regard to economic characteristics is proposed. Due to the fact that, as a rule, it is not possible to prevent sudden failures of the elements in the process of diagnosis, it is necessary to repair or replace the elements routinely through the optimal inter-repair work. Such failures include not diagnosed parts and joints.

In this regard, it is necessary to be guided by the following:

1. With regard to sudden failures, all permissible deviations of the parameters - DOR, DMR, DCR are equated to unity.

2. Economic indicators: the costs of diagnosis - B, as well as continuous costs $-\mathrm{C}_{\mathrm{H}}$ are equated to zero. 
3. The costs characterized by the sum of products of the probability of failure and costs caused by a failure for different periodicity - LOR, LMR, LCR and the probability of preventive replacement for the corresponding costs are determined by the formula:

$$
G_{\min }=\frac{C_{H} \int_{0}^{L} f(l) d l+C_{n} \int_{L}^{\infty} f(l) d l}{\int_{0}^{L} f(l) d L+L \int_{L}^{\infty} f(l) d l},
$$

where: $f(1)$ - density distribution of the object's operating time before failure.

The denominator of expression (2) is the average actual operating time of suddenly failed elements, regularly replaced at the time $i \cdot L$.

In case when the Weibull law of distribution is considered, the displacement parameter - $t_{C}$

4. By the lowest value ${ }^{G_{i}}$, the optimum operating time before the regular replacement of the elements is established $-L_{1}^{O P T}$.

Algorithms (1) and (2) are oriented to objects whose resources are limited by "sudden" failures, while objects that have not failed during the run period from 0 to $\mathrm{L}$ are subject to scheduled repair in a given amount. In addition, it is assumed that with the restoration of failure and with scheduled repair, the same degree of resource recovery is achieved.

In practice, most assembly units are subject not only to sudden but also to gradual failures arising from the consequent increase in the amount of wear, reduction in resistance, elasticity of elements, fatigue strength, etc. The model of gradual failures has characteristic features. Figure 3 shows its graphical interpretation: a set of identical i-elements wears out with a constant intensity $\lambda \mathrm{i}$ until the wear limit value is reached - Un. If these elements are not restored before reaching the operating time $\mathrm{t} 3$, then all of them will fail, i.e. their wear will reach or exceed the value Un.

Fig. 3 shows the distribution of the frequency of gradual failures of the considered set of elements in the coordinates $\varphi$ and $t$. This distribution is placed in the operating range from $\mathrm{Un} / \lambda \max$ to $\mathrm{Un} / \lambda \min$.

If everything is subject to control with a periodicity of $1 \mathrm{~m}$, and those of them whose wear exceeds the value of $\mathrm{D}$ replaced by elements with a controlled value equal to $\mathrm{D}$ (tolerance), then the number of gradual failures will be reduced. In Fig. 3, the proportion of the shaded areas from the total area outlined by the distribution curve $\varphi(t)$ characterizes the degree of decrease in the number of failures.

Obviously, with a decrease in LM to a value equal to (Un-D)/ $/ 2 \max$, all gradual failures can be prevented, but the costs for frequent execution of scheduled replacements of elements with wear exceeding $\mathrm{D}$. The number of scheduled replacements is reduced when increasing the value of Un-D, i.e. when assigning a more "hard" tolerance. Thus, this model illustrates the possibility of using the value $\mathrm{D}$ of the tolerance assigned during repair as one of the factors controlling the number of gradual failures. 


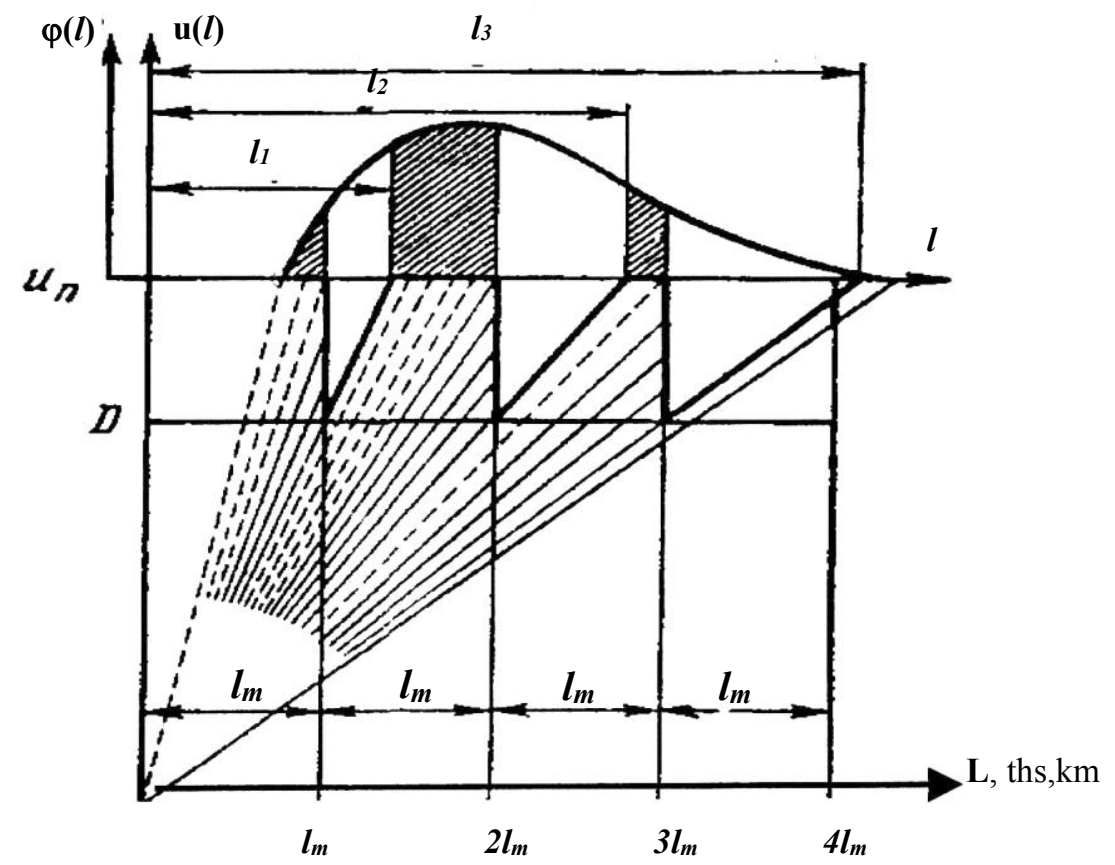

Fig. 3. The model of gradual failures.

It should be noted that for $\mathrm{D}=\mathrm{Un}$, the probability of gradual failure in relation to the scheme in Fig. 3 is defined as:

$$
q_{i}\left(D, l_{M}\right)=\int_{i-1}^{i L_{M}} \varphi(l) d l
$$

where: I - ordinal number of scheduled repair.

The target function of optimizing the values of LM and D in this case:

$$
G=\min \left\{\frac{A \cdot Q\left(D_{0}, L_{M}\right)+\left[1-Q\left(D_{0}, L_{M}\right)\right]+B+N_{H}\left(D_{0}, L_{M}\right)+S\left(D_{0}, L_{M}\right)}{T_{0}\left(D_{0}, L_{M}\right)}\right\},
$$

where: A, B - average values of discrete costs - respectively, to restore the required value of the monitored parameter, including replacement of the element and its verification (when using diagnostic tools) - represented in the shares of costs to eliminate the consequences of gradual failures;

D0 - deviation of the admissible value of the monitored parameter from its limit value (D0=D0/Un);

Q (D0, LM) - the probability of failure, i.e. achievement of Un, during the service life of the object, as a function of set values and operating time before the repair (LM);

$\mathrm{Nn}(\mathrm{D} 0, \mathrm{LM})$ - number of checks of the monitored parameter - during the service life of the element - as a function of the variable (assigned) values D0 and LM;

T0 (D0, LM) - the average value of the resource used for different versions of D0 and LM.

The model under consideration is rather arbitrary. In general, the dependence of $\lambda$ on the operating time is nonlinear, the value of Un at which the elements fail is not the same for each of them, i.e. Un=f(i). Instead of tolerance D, the "tolerance range" (from Dmin to Dmax) 
is often used for repairs, some of the elements after the failure are not restored but are subject to be written off. In practice, the cost of eliminating the gradual failure is also not a constant value. Taking into account the above circumstances, a more universal algorithm of the target function has been formed to determine the optimum values of the standard operating time of the assembly unit that is subject to both gradual and sudden failures in operation:

$$
G=\min \left[\frac{C_{0}+A \cdot H_{G}(y)+A^{*} \cdot H_{S}(y)+C \cdot H_{Z}(y)+B \cdot H_{K}(y)+H_{C} P_{C}}{T_{W}}+C_{H}\right],
$$

where: $y$ - functional of $\alpha, \mathrm{VZ}, \mathrm{DR}, \mathrm{DOR}, \mathrm{DMR}, \mathrm{DCR}, \mathrm{U}, \mathrm{KB}, \mathrm{PH}, \mathrm{P} 0, \mathrm{~PB}, \mathrm{PS}, \mathrm{TCR}, \mathrm{TMR}$, TOR, TW, T0, qu', qu",qMR, qOR, qW.

In this case: $\alpha, \mathrm{VZ}, \mathrm{DR}$ - the indices of the degree of "non-smoothness" of the deviation function of the value of the controlled parameter of the assembly unit and the indicator that takes into account the existence of a "running-in" period, respectively;

$\mathrm{D}$ - the allowable deviation of the parameter (indices CR, MR, and OR correspond to types of repair for which the deviation is normalized);

$\mathrm{U}$ - the limiting value of the deviation of the parameter;

$\mathrm{KB}$ - the degree of restoration of the normative value of the parameter when repairing the assembly unit;

$\mathrm{PH}$ - probability of replacement of an element (details, joints of details) of an assembly unit by a new one;

$\mathrm{P} 0$ - probability of detection of failure of the assembly unit in the period between controls;

$\mathrm{PB}$ - probability of writing-off the assembly unit when the limiting value of the monitored parameter is reached;

PC - probability of a second-kind error in diagnosing the state of the assembly unit, i.e. continued operation of the object with an erroneous diagnosis of the loss of its operability;

TCR, TMR, TOR - average value of the operating time of the assembly unit before CR, $\mathrm{MR}, \mathrm{OR}$;

TW, T0 - average value of operating time before write-off (by the controlled parameter);

qu', qu', qMR, qOR, qW - coefficients of variation of the limiting value of the monitored parameter (causing gradual and sudden failures, respectively), operating time before scheduled repairs $\mathrm{CR}, \mathrm{MR}$, and $\mathrm{OR}$;

$\mathrm{C} 0, \mathrm{HC}$ - average values of expenses for acquisition and write-off of the assembly unit;

A, B, C - the average costs of eliminating gradual and sudden failures (including costs associated with violation of the established operating mode in case of failures) for scheduled maintenance and for equipment diagnostics (control);

$\mathrm{H}$ - number of recoveries (indices $\mathrm{G}, \mathrm{S}, \mathrm{Z}, \mathrm{K}$ correspond to recoveries after gradual, sudden failures during scheduled repair by results of diagnosing).

This algorithm (5) is adapted to the repair cycle consisting of three types of repair - the current CR, the medium MR, and the overall OR. Input control of the condition of the assembly unit with the use of diagnostic tools is joined with CR. The algorithm can be implemented initially to each of the constituent parts of the assembly unit separately, and then, from the local optimums found, the global optimum of the mileage norms and the tolerances before scheduled repairs for the assembly unit as a whole is determined. A similar procedure is envisaged for finding the required values for the complete set of locomotive assembly units. At the same time, a minimum of expenses for the sum of the functionals found for the units and components is determined, which makes it possible to establish the values of $\mathrm{T}$ and $\mathrm{D}$ corresponding to this minimum. The use of the proposed model allows determining the terms of scheduled repairs, to form the lists of repair works for these terms, i.e. form repair types, as well as a system of tolerances for controlled parameters for each of them. In a particular case, this algorithm can be used to determine the optimum tolerance for 
the parameters of individual elements at specified locomotive run rates between scheduled repairs.

\section{Discussion}

At present, a scheduled preventive maintenance system for locomotives is applied on the railway network. This is due to the construction of locomotives and the insufficiently developed infrastructure of locomotive depots. At the same time, the main direction in the design of innovative locomotives is the organization of repairs taking into account the technical condition of the equipment. For this, it is necessary to improve the systems of technical diagnostics and software systems for managing the technical condition of the locomotive. The application of the algorithm of the target function of the overhaul runs taking into account the gradual and sudden failures of locomotive equipment makes it possible to choose the most rational repair system with a repair cycle at minimum costs. The research conducted on the basis of the computer analytical package "Statistica" allowed carrying out statistical modeling of random and other quantities, optimizing time between overhauls, allowable values of equipment parameters for current, medium, and overall repairs of equipment. The results of the research have been repeatedly reported on the scientific and technical councils of the All-Russian Railway Transport Research Institute and are used in the development of technical requirements for innovative locomotives.

\section{Conclusion}

The considered models and algorithms allow improving the way of managing the technical condition of the locomotive fleet by optimizing the repair cycle, the standards of the time between overhauls, the regulated structure of scheduled repairs, and the tolerances for the technical parameters of the assembly units that are implemented in the performance of these works with given level of operational reliability of the traction rolling stock. The developed mathematical model and algorithm for controlling the technical state of the equipment of an electric locomotive with the use of the target function of optimizing the repair cycle will allow increasing the failure-free operation of the main units and components of an electric locomotive with minimal costs for repairs. The use of the optimization algorithm for the repair cycle of locomotives is fully integrated into innovative control technologies for repair based on the blockchain platform.

\section{References:}

1. Augmented technology lab opens, Railway Gazette International 12, 55 (2017)

2. Hamamatsu revamp keep Tokaido ShinKansen fleet fit., Railway Gazette International 12, 34-35 (2017)

3. V.M. Mikhlin, A.T. Osyaev, Machine and technological station 15, 26-33 (2002)

4. Ch. Muller, Der Eisenbahningenier 6, 50-53 (2017)

5. A.T. Osyaev, I.P. Isaev, A.V. Gorsky Bulletin of the All-Union Scientific Research Institute of Railway Transport 6, 31 (1991)

6. A.T. Osyaev, A.N. Kondratenko, Railway transport 1, 24-26 (1989)

7. A.T. Osyaev, S.A. Shuleshko, Integrated system of maintenance and repair of locomotives with the use of innovative technologies: a training manual (IMO ROAT,Moscow, 2017) 
8. A.Sh. Rabinovich, A.A. Seltzer, A.A. Sharovskii, Methodical instructions on the estimation, forecasting, and normalization of the resource and the reliability of agricultural equipment (GOSNITI, Moscow, 1975)

9. Guterwagen-Monitoring-Sistem, RailWatch-fleight-wagon monitoring system, Signal + Draht 9, 78 (2017)

10. D. Rees, Eisenbahn techishe Rundschau 6, 30 (2017)

11. N.N. Smirnov, Methodology of operational control of the efficiency of the process of technical operation of aircraft in operational aviation enterprises (Transport, Moscow, 1985)

12. Siemens vernetzt Lokomotiven der DB Cargo, Siemens addiding networking to DB Cargo locos, Signal + Draht 9, 77 (2017)

13. R. Surber, St. Hoffman, Der Eisenbahningenier 6, 38-40 (2017)

14. J. Villegoureix, Chemins de Fer 567, 28-32 (2017) 